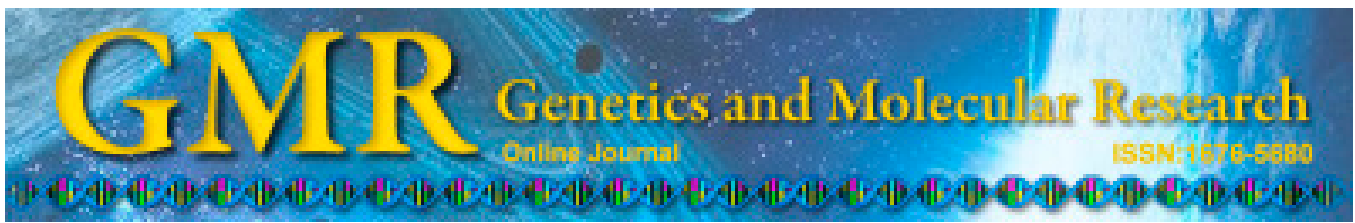

\title{
Development and characterization of microsatellite markers via cross-species amplification of Paramisgurnus dabryanus
}

\author{
G. Chen, S. Huang, J. Gao, X. Bai, W. Wang and X. Cao \\ College of Fisheries, Key Laboratory of Freshwater Animal Breeding, \\ Ministry of Agriculture, Huazhong Agricultural University, Wuhan, Hubei, \\ China
}

Corresponding author: X. Cao

E-mail: caoxiaojuan@mail.hzau.edu.cn

Genet. Mol. Res. 14 (2): 5694-5698 (2015)

Received January 25, 2013

Accepted April 29, 2015

Published May 29, 2015

DOI http://dx.doi.org/10.4238/2015.May.29.1

\begin{abstract}
The large-scale loach, Paramisgurnus dabryanus, is a small freshwater fish of major economic importance in many Asian countries, particularly China and South Korea. Fifteen polymorphic microsatellite (simple sequence repeat) markers were obtained through cross-species amplification between this loach and a related species, Misgurnus anguillicaudatus (GenBank accession numbers: $\mathrm{KC} 117456$ to $\mathrm{KC117470)}$. The number of alleles per locus ranged from 5 to 12 among 40 individuals, and the average observed and expected heterozygosities were 0.344 and 0.828 , respectively. Three loci showed significant deviations from Hardy-Weinberg equilibrium. These polymorphic loci could provide a valuable tool for investigations of the population genetics, phylogeography, and conservation genetics of $P$. dabryanus.
\end{abstract}

Key words: Paramisgurnus dabryanus; Microsatellite markers; Cross-amplification; Polymorphism 


\section{INTRODUCTION}

The large-scale loach, Paramisgurnus dabryanus, is a loach species that is distributed widely across East Asia, from Russia to China. Because of its high economic and medicinal value, this loach is widely cultured in China and South Korea (You et al., 2009). Recently, the wild population of $P$. dabryanus has substantially decreased due to overfishing and water pollution. Research of this loach has mostly focused on its breeding physiology (You et al., 2008), growth (Wang et al., 2009), development (Zhang et al., 2001), and toxicology (Li et al., 2003). The genetic diversity, population structure, and molecular markerassisted breeding of this species are currently poorly understood, primarily due to a lack of variable genetic markers.

Microsatellites have become one of the most popular molecular markers that are used in a wide variety of genetic investigations (Russell et al., 1997; Stevanato et al., 2013). There are several methods for microsatellite isolation (Zane et al., 2002). In contrast, to isolate microsatellites from target species de novo, cross-species amplification is a cost-effective method resulting in the rapid development of microsatellites from closely related taxa (Schlotterer et al., 1991; Zheng et al., 1995, Gu et al., 2012). To date, only six microsatellite sequences of $P$. dabryanus have been reported in a single study (You et al., 2012). Therefore, screening for more polymorphic microsatellites in this loach is very important and necessary for future genetic investigations. In this study, we obtained 15 polymorphic microsatellites for P. dabryanus from a related species, Misgurnus anguillicaudatus, through cross-species amplification.

\section{MATERIAL AND METHODS}

In order to characterize isolated microsatellites, 40 P. dabryanus individuals were collected from Hubei (23 individuals) and Henan (17 individuals) provinces, China, in 2012. Fin samples were dissected from these loaches and stored in 100\% ethanol immediately after removal. Genomic DNA was extracted from fins following the method described in Lassner et al. (1989), which was further modified by Torres et al. (1993).

M. anguillicaudatus, which is a species related to $P$. dabryanus, was used in this study to obtain microsatellites by cross-species amplification. Sixty-three pairs of microsatellite primers from M. anguillicaudatus (Table 1, Morishima et al., 2008; DDBJ database) were randomly selected and synthesized for polymerase chain reaction (PCR) amplification. PCR was performed in a $10-\mu \mathrm{L}$ reaction mixture containing 8-40 ng DNA (a mixed DNA sample of six P. dabryanus individuals), 1 X PCR buffer (10 mM Tris- $\mathrm{HCl}, \mathrm{pH} 8.3,1.5 \mathrm{mM} \mathrm{MgCl}, 50 \mathrm{mM}$ $\mathrm{KCl}$ ), $120 \mu \mathrm{M}$ dNTPs, $0.15 \mu \mathrm{M}$ of each primer, and $1 \mathrm{U}$ Taq DNA polymerase (all reagents were purchased from Dingguo Bio., Beijing, China). Amplification was achieved in a T $100^{111}$ thermal cycler and the conditions were as follows: $94^{\circ} \mathrm{C}$ for $5 \mathrm{~min}, 94^{\circ} \mathrm{C}$ for $40 \mathrm{~s}$, annealing temperature $\left(53.1^{\circ}-62.2^{\circ} \mathrm{C}\right.$; efforts were made to optimize the annealing temperature) for $45 \mathrm{~s}$, $72^{\circ} \mathrm{C}$ for $50 \mathrm{~s}$ for 35 cycles, with a final step of $10 \mathrm{~min}$ at $72^{\circ} \mathrm{C}$. PCR products were checked by $1.5 \%$ agarose gel electrophoresis and visualized under a UV transilluminator after ethidium bromide staining. Based on the results of agarose gel electrophoresis, PCR products with clear bands and reasonable sizes were selected and then sequenced by the Shanghai ShengGong Biological Engineering Technology \& Services Co., Ltd., Shanghai, China to ensure that the products were genuine microsatellites. After filtering out the 63 microsatellite loci with poor 
cross-amplification, combined with the sequencing results, the remaining loci (genuine microsatellites) were tested in 40 P. dabryanus individuals for polymorphism analyses. The PCR products were separated on $6 \%$ non-denaturing polyacrylamide gel. Gels were then stained with $1 \mathrm{~g} / \mathrm{L}$ silver nitrate in water and visualized under ultraviolet light. The size of alleles was estimated using a 501 bp DNA marker (PUC18DNA/msp1) combined with image analysis, as described by Nelson et al. (1998). The number of alleles $\left(N_{\mathrm{A}}\right)$, observed $\left(H_{\mathrm{O}}\right)$ and expected $\left(H_{\mathrm{E}}\right)$ heterozygosity, and departure from Hardy-Weinberg equilibrium (HWE) were estimated in POPGENE version 1.32 (Yeh et al., 1999).

Table 1. Names of 63 microsatellites loci of Misgurnus anguillicaudatus and their DDBJ accession numbers.

\begin{tabular}{llllllllll}
\hline Locus & $\begin{array}{l}\text { DDBJ } \\
\text { Accession No. }\end{array}$ & Locus & $\begin{array}{l}\text { DDBJ } \\
\text { Accession No. }\end{array}$ & Locus & $\begin{array}{l}\text { DDBJ } \\
\text { Accession No. }\end{array}$ & Locus & $\begin{array}{l}\text { DDBJ } \\
\text { Accession No. }\end{array}$ & $\begin{array}{l}\text { Locus } \\
\text { DDBJ } \\
\text { Accession No. }\end{array}$ \\
\hline Mac2 & AB060172 & Mac48 & AB081626 & Mac167 & AB303453 & Mac361 & AB303496 & Mac497 & AB303578 \\
Mac11 & AB060181 & Mac49 & AB060186 & Mac190 & AB303455 & Mac364 & AB303498 & Mac519 AB303584 \\
Mac21 & AB081619 & Mac50 & AB060187 & Mac229 & AB303459 & Mac375 & AB303500 & Mac523 AB303585 \\
Mac23 & AB081621 & Mac51 & AB081627 & Mac235 & AB303461 & Mac387 & AB303506 & Mac532 & AB303586 \\
Mac24 & AB060178 & Mac60 & AB081631 & Mac242 & AB303463 & Mac404 & AB303512 & Mac541 AB303588 \\
Mac25 & AB060179 & Mac62 & AB081632 & Mac262 & AB303468 & Mac408 & AB303514 & Mac559 AB303592 \\
Mac31 & AB081622 & Mac63 & AB081633 & Mac264 & AB303470 & Mac425 & AB303516 & Ma574 AB303594 \\
Mac36 & AB060180 & Mac103 & AB303436 & Mac276 & AB303473 & Mac429 & AB303518 & Mac576 AB303595 \\
Mac37 & AB060181 & Mac105 & AB303437 & Mac277 & AB303474 & Mac449 & AB303519 & Mac605 AB303605 \\
Mac39 & AB081623 & Mac123 & AB303441 & Mac291 & AB303477 & Mac456 & AB303522 & Mac612 AB303607 \\
Mac43 & AB081624 & Mac133 & AB303444 & Mac293 & AB303478 & Mac462 & AB303524 & Mac627 AB303610 \\
Mac45 & AB060185 & Mac140 & AB303445 & Mac320 & AB303483 & Mac466 & AB303525 & & \\
Mac47 & AB081625 & Mac165 & AB303451 & Mac331 & AB303487 & Mac477 & AB303575 & &
\end{tabular}

\section{RESULTS AND DISCUSSION}

Of the 63 microsatellite loci from M. anguillicaudatus, 39 showed no amplification products in $P$. dabryanus, while 6 presented very weak and multiple bands, which were not scorable. Hence, 18 pairs of primers resulted in suitable and scorable PCR products in $P$. dabryanus. After sequencing these PCR products, 15 loci were verified to be microsatellites. The 15 microsatellite sequences were submitted to GenBank (accession numbers KC117456 to KC117470). Characteristics of the 15 microsatellite loci in $P$. dabryanus are listed in Table 2 . All of the 15 loci were highly polymorphic with an average of 9.07 alleles per locus (ranging from 5 to 12). The average observed and expected heterozygosities were 0.344 and 0.828 , respectively. Three loci were found to deviate from HWE after Bonferroni correction $(\mathrm{P}<0.05)$.

The present study successfully demonstrated cross-species amplification of microsatellite primers in Cobitidae (15/63;23.8\%), confirming previous studies in Phasianidae (Gu et al., 2012), Muridae (Sun at al., 2009), and Serranidae (Dong et al., 2008), which all showed that cross-species amplification was a very effective method for microsatellites development. These results might suggest that many microsatellites and their flanking regions are relatively conserved in Cobitidae, which would facilitate the construction of a syntenic map in this family. Adequate levels of variation at the 15 microsatellite loci suggest that these markers would be potentially useful for studies on parentage and kinship analysis, population genetics, and phylogeography in $P$. dabryanus. 
Paramisgurnus dabryanus microsatellite markers

5697

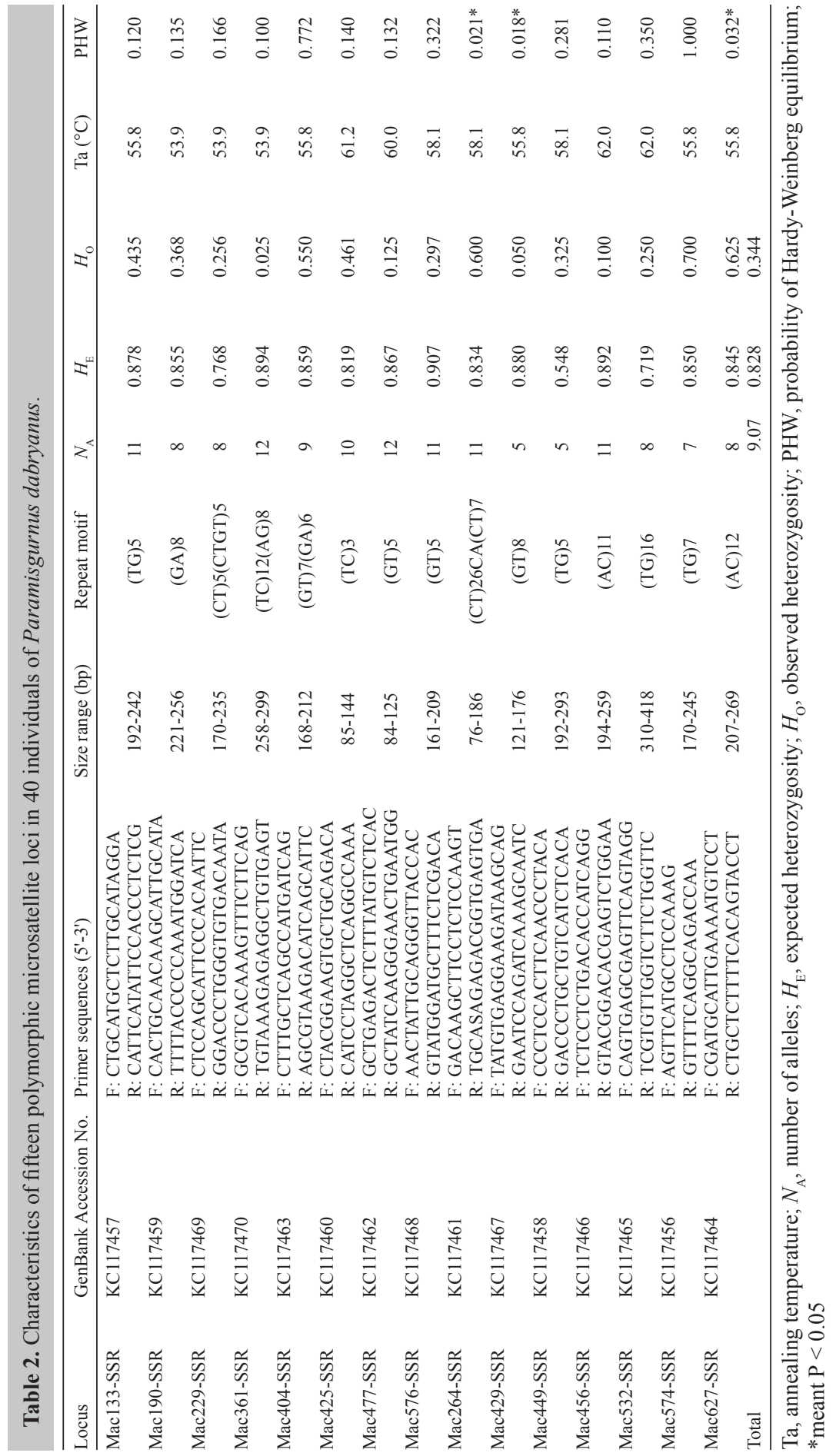




\title{
ACKNOWLEDGMENTS
}

\author{
Research supported by the National Natural Science Foundation of China (\#31201719) \\ to Xiaojuan Cao.
}

\section{REFERENCES}

Dong Y, Han J and Cai H (2008). Cross-species amplification and characterization of polymorphic microsatellite loci in Hong Kong grouper, Epinephelus Akaara. J. Beijing Normal Univ. (Nat. Sci.) 44: 511-514.

Gu L, Liu Y, Wang N and Zhang Z (2012). A panel of polymorphic microsatellites in the Blue Eared Pheasant (Crossoptilon auritum) developed by cross-species amplification. Chin. Birds 3: 103-107.

Lassner MW, Peterson P and Yoder JI (1989). Simultaneous amplification of multiple DNA fragments by polymerase chain reaction in the analysis of transgenic plants and their progeny. Plant Mol. Biol. Rep. 7: 116-128.

Li XY, Liu YD, Song LR, Lei LM et al. (2003). The toxicity of microcystins to embryo and larvae of loach (Paramisgurnus dabryanus Sauvage). Acta Hydrobiol. Sin. 3: 318-319.

Morishima K, Nakayama I and Arai K (2008). Genetic linkage map of the loach Misgurnus anguillicaudatus (Teleostei: Cobitidae). Genetica 132: 227-241.

Nelson RJ, Beacham TD and Small MP (1998). Microsatellite analysis of the population structure of a vancouver island sockeye salmon (Oncorhynchus nerka) stock complex using nondenaturing gel electrophoresis. Mol. Mar. Biol. Biotechnol. 7: 312-319.

Russell JR, Fuller JD, Macaulay M, Hatz BG, et al. (1997). Direct comparison of levels of genetic variation among barley accessions detected by RFLPs, AFLPs, SSRs and RAPDs. Theor. Appl. Genet. 93: 714-720.

Schlotterer C, Amos B and Tautz D (1991). Conservation of polymorphic simple sequence in cetacean species. Nature 354: 63-65.

Stevanato P, Trebbi D, Biancardi E, Cacco G, et al. (2013). Evaluation of genetic diversity and root traits of sea beet accessions of the Adriatic Sea coast. Euphytica 189: 135-146.

Sun B, Bao Y, Zhang L, Zhao Q et al. (2009). Cross-species amplification of rat and mouse microsatellite DNA Loci in the Niviventer confucianus. Chin. J. Zool. 44: 145-150.

Torres AM, Weeden NF and Martin A (1993). Linkage among isozyme, RFLPs and RAPD markers in Vicia faba. Theor. Appl. Genet. 85: 937-945.

Wang K, Ling QF, Li Q, Cheng F, et al. HJ (2009). Primary study on the age and growth of Misgurnus anguillicaudatus and Paramisgurnus dabryanus in the area of Suzhou. J. Shanghai Ocean Univ. 18: 163-167.

Yeh FC, Yang R and Boyle T (1999). Popgene version 1.32. Microsoft windows-based freeware for population genetic analysis. University of Alberta, Edmonton, Alberta.

You C, Tong J and Yu X (2012). Microsatellite DNA analysis on genetic diversity of seven populations of Paramisgurnus dabryanus. J. Hydroecol. 33: 84-91.

You CH, Yu XM, Tan DQ and Tong JG (2008). Gynogenesis and sex determination in large-scale loach Paramisgurnus dabryanus (Sauvage). Aquacult. Int. 16: 203-214.

You CH, Yu XM and Tong JG (2009). Detection of hybridization between two loach species (Paramisgurnus dabryanus and Misgurnus anguillicaudatus) in wild population. Environ. Biol. Fish. 86: 65-71.

Zane L, Bargelloni L and Patarnello T (2002). Strategies for microsatellite isolation: a review. Mol. Ecol. 11: 1-16.

Zhang HY (2001). The embryonic development and fingerling culture of loach, Paramisgurnus dabryanus Sauvage. Acta Hydrobiol. Sin. 31: 55-62.

Zheng W, Stacey NE, Coffin J and Strobeck C (1995). Isolation and characterization of microsatellite loci in the goldfish Carassius auratus. Mol. Ecol. 4: 791-792. 